\section{The seroprevalence of SARS-CoV-2 in patients on haemodialysis}

\author{
Viyaasan Mahalingasivam (1) and Laurie Tomlinson (1)
}

A new study reports the seroprevalence of SARS-CoV-2 antibodies among a cross-section of patients on haemodialysis and uses these data to estimate seroprevalence in the general US population. Although this study demonstrates the potential of monitoring infectious disease prevalence in dialysis populations, the findings should be interpreted with caution.

Refers to Anand, S. et al. Prevalence of SARS-CoV-2 antibodies in a large nationwide sample of patients on dialysis in the USA: a cross-sectional study. Lancet 396, 1335-1344 (2020).
Epidemiological research into SARS-CoV-2 is challenging. A combination of regional differences, variations in symptoms among infected people and a limited (but increasing) capacity to undertake swab testing and focused screening in groups such as carers in nursing homes, prevalence of infection. Clear insights into many questions have been slow to emerge; for example, the factors that are associated with being infected with SARS-CoV-2 as opposed to dying of COVID-19, which is sadly easier to measure. Large, population-representative cohorts with detailed demographic and health information sampled by experienced technicians at multiple time points are needed. Some studies, such as the UK REACT study ${ }^{1}$ and ENE-COVID in Spain ${ }^{2}$, go a long way to meeting these requirements, but all have limitations. Antibody testing offers a different insight - evidence of previous infection with SARS-CoV-2. Well-designed seroprevalence studies are critical to determine the proportion of a population who have been infected to help predict future infection dynamics and guide public health policy.

\section{$8.0 \%$ of tested patients} were seropositive for SARS-CoV-2 antibodies

A new study uses a pragmatic approach to define the prevalence of previous infection by testing for antibodies to SARS-CoV-2 in routinely collected blood from randomly sampled patients on haemodialysis across the USA ${ }^{3}$. These patients already undergo has made it difficult to understand the true blood tests every month to assess dialysis adequacy and management of kidney failure complications, so their blood can be tested for SARS-CoV-2 antibodies without additional venepuncture. This approach mitigates bias through non-response that would be expected for studies requiring blood testing in the general population. In addition, dialysis centres are incentivized to undertake routine monthly blood testing, ensuring very high data completeness. The study was funded by Ascend Clinical, a commercial laboratory that processed the tests using a high-sensitivity, high-specificity chemiluminescence assay. The laboratory receives specimens from approximately 63,000 patients in around 1,300 dialysis facilities; this cohort comprised the source population.

As the patients on dialysis were older and more likely to be Black than the US general adult population, the researchers standardized their seroprevalence estimates to this

\section{This estimate suggests}

that over $90 \%$ of the US adult population could be susceptible to SARS-CoV-2

population. They then correlated their results with publicly available state-level population measures of cases, deaths and testing, as well as county-level measures in California, New York and Texas, which had the highest densities of participants. More than 31,000 patients were systematically sampled, of whom $>28,000$ were tested in July 2020. The age and sex distributions of the sampled population were fairly similar to the US adult dialysis population although patients from the West were overrepresented and those from the Midwest were underrepresented. Race and ethnicity was unknown in $40 \%$ of patients; as a proxy, patient-level residence data was linked to ZIP-code tabulation area (ZCTA) data from the 2018 American Community Survey to identify each patient's majority neighbourhood race and ethnicity.

The study reports that $8.0 \%$ of tested patients were seropositive for SARS-CoV-2 antibodies (TABLE 1). Using ZCTA data, the adjusted risk of seropositivity was highest among non-Hispanic Black and Hispanic and Black patients and was lowest among nonHispanic white patients. The risk was also increased by poverty and living in densely populated and urbanized areas. These findings are consistent with the results of international research in the general population. When standardized to the US adult population using weighted stratification by census region, age and sex, seropositivity was estimated to be $9.3 \%$. This estimate suggests that over $90 \%$ of the US adult population could be susceptible to SARS-CoV-2, which is alarming considering the scale of impact on the country so far but is consistent with findings from England ${ }^{1}$ and Spain ${ }^{2}$.

\begin{tabular}{|c|c|c|c|}
\hline \multirow{2}{*}{$\begin{array}{l}\text { ZCTA majority } \\
\text { race and } \\
\text { ethnicity }\end{array}$} & \multicolumn{3}{|c|}{ SARS-CoV- 2 antibody seropositivity ( $\%$ ( $95 \%$ confidence interval)) } \\
\hline & $\begin{array}{l}\text { Sampled dialysis } \\
\text { population } \\
(n=28,503)\end{array}$ & $\begin{array}{l}\text { Standardized } \\
\text { estimate for US } \\
\text { dialysis population } \\
(n=499,150)\end{array}$ & $\begin{array}{l}\text { Standardized estimate } \\
\text { for US adult population } \\
(n=253,815,197)\end{array}$ \\
\hline Hispanic & $9.0(8.2-10.0)$ & $9.4(8.5-10.3)$ & $11.3(9.8-12.9)$ \\
\hline Hispanic and Black & $14.6(13.3-16.1)$ & $14.5(13.2-15.9)$ & $16.3(14.3-18.5)$ \\
\hline Non-Hispanic Black & $14.7(13.3-16.3)$ & $14.1(12.9-15.5)$ & $13.9(12.1-16.0)$ \\
\hline Non-Hispanic white & $4.2(3.8-4.7)$ & $4.3(3.8-4.7)$ & $4.8(4.1-5.5)$ \\
\hline Other & $7.3(6.8-7.9)$ & $8.0(7.4-8.6)$ & $8.9(8.0-9.8)$ \\
\hline Overall & $8.0(7.7-8.4)$ & $8.3(8.0-8.6)$ & $9.3(8.8-9.9)$ \\
\hline
\end{tabular}

ZCTA, ZIP-code tabulation area. Data obtained from REF. ${ }^{3}$. 
In addition, the research identified striking variation in seroprevalence, ranging from $0 \%$ in seven states to $34 \%$ in New York state, consistent with the substantial outbreak in the city ${ }^{4}$. State seroprevalence correlated with cumulative deaths and cases per 100,000 population and the odds of seropositivity were lower in areas where workplace visits were reduced before nationwide restrictions were implemented. These findings offer the tantalizing prospect of using seroprevalence to provide feedback on policy changes but equally might result from an ecological fallacy. For example, patients on haemodialysis might have undertaken strict shielding measures to protect themselves if they lived in regions with rapidly rising infection rates, which also triggered policy changes such as a reduction in workplace visits.

The idea of conducting nationwide serological surveillance through a randomly sampled sentinel population of patients on haemodialysis is an attractive proposition. However, concerns exist about whether conclusions for the general population can be reliably derived from the haemodialysis population even when standardization methods are used. Patients on dialysis are subject to increased risk of infection due to three times weekly visits to haemodialysis facilities, which often involve the use of shared patient transport and close contact with other patients despite infection control measures. In addition, patients with kidney failure often have high levels of comorbidity and frailty, leading to increased risk of transmission through contact with carers. Outbreaks of COVID-19 within haemodialysis units have been reported worldwide ${ }^{5}$.

On the other hand, seroprevalence in the general population could potentially be underestimated using data from patients on dialysis. Mortality from COVID-19 among patients on haemodialysis is higher than in the general population, resulting in survivor bias. The latest report from the UK Renal registry, which includes data from $>2,700$ patients on haemodialysis with COVID-19, reported a mortality of $22 \%{ }^{6}$, which is remarkably consistent with reports from other countries ${ }^{5}$. In addition, patients on dialysis are likely to take greater precautions with physical distancing measures than the general population due to their increased risk, so may be at lower risk of community transmission. Lastly, patients on haemodialysis generate a weaker immune response to stimuli such as the Hepatitis B vaccine ${ }^{7}$ and so may be less likely to seroconvert than the general population. Although a recent study demonstrated $97 \%$ seroconversion in patients with kidney failure and previously confirmed SARS-CoV-2 infection ${ }^{8}$, similar to rates seen in the general population $^{9}$, the comparative proportion who seroconvert after asymptomatic infection remains unknown. Before a strategy of serological surveillance of patients on haemodialysis could be adopted to guide wider public health policy, longitudinal antigen screening studies would be needed to better understand asymptomatic seroconversion in these patients. In addition, practical difficulties exist. The rapid results and information flow as well as the likely consistency of testing methods in the current study were achieved because the antibodies were measured at a central laboratory specializing in blood tests for patients with kidney failure; these advantages would not translate to blood samples being measured at local hospital laboratories in countries such as the UK.

This research shows the huge potential of monitoring infectious disease prevalence in dialysis populations but given the intensity of interest in SARS-CoV-2 seroprevalence for policymaking and public health messaging, little room exists for speculation. The clinical utility of serological surveillance for SARS-CoV-2 in patients on haemodialysis is uncertain and the duration of antibody response as well as the extent to which humoral and cellular processes confer protection from reinfection remain unknown with recent reports of waning seroprevalence over time ${ }^{10}$. However, at the very least, surveillance provides invaluable information on asymptomatic cases of SARS-CoV-2 infection in patients on haemodialysis to better understand local outbreaks and guide improvements for infection prevention in this extremely vulnerable patient group.

\section{Viyaasan Mahalingasivam (D') and Laurie Tomlinson $\left(\mathbb{D}^{2 凶}\right.$ \\ 'Department of Renal Medicine, Barts Health NHS Trust, London, UK. \\ ${ }^{2}$ Department of Non-Communicable Disease Epidemiology, London School of Hygiene and Tropical Medicine, London, UK.

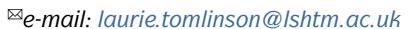 https://doi.org/10.1038/s41581-020-00379-y}

1. Ward, H. et al. Antibody prevalence for SARS-CoV-2 in England following first peak of the pandemic: REACT 2 study in 100,000 adults. medRxiv https://doi.org/10.1101/2020.08.12.20173690 (2020).

2. Pollán, M. et al. Prevalence of SARS-CoV-2 in Spain (ENE-COVID): a nationwide, population-based seroepidemiological study. Lancet 396, 535-544 (2020).

3. Anand, S. et al. Prevalence of SARS-CoV-2 antibodies in a large nationwide sample of patients on dialysis in the USA: a cross-sectional study. Lancet 396 , 1335-1344 (2020).

4. Rosenberg, E. S. et al. Cumulative incidence and diagnosis of SARS-CoV-2 infection in New York. Ann. Epidemiol. 48, 23-29 (2020).

5. Hiremath, S. et al. Dialysis and CKD-summary of mortality from COVID-19 among dialysis patients. \#NephJC http://www.nephjc.com/ news/2020/3/23/covid-and-the-kidney-dialysis-edition (2020)

6. The Renal Association. COVID-19 surveillance report for renal centres in the UK. The Renal Association https://renal.org/sites/renal.org/files/ALL_REGIONS CENTRES covid report 21102020.pdf (2020).

7. Bel'eed, K., Wright, M., Eadington, D., Farr, M. \& Sellars, L. Vaccination against hepatitis B infection in patients with end stage renal disease. Postgrad. Med. J. 78, 538-540 (2020).

8. Clarke, C. et al. High prevalence of asymptomatic COVID-19 infection in hemodialysis patients detected using serologic screening. J. Am. Soc. Nephrol. 31, 1969-1975 (2020).

9. Wajnberg, A. et al. Humoral response and PCR positivity in patients with COVID-19 in the New York City region, USA: an observational study. Lancet Microbe. 1, 283-289 (2020).

10. Alford, J. Coronavirus antibody prevalence falling in England, REACT study shows. Imperial College London https://www.imperial.ac.uk/news/207333/ coronavirus-antibody-prevalence-falling-england-react/ (2020).

Competing interests

The authors declare no competing interests. 